Pädiatrie und Pädologie

Supplementum 6

\title{
Kind und Umwelt
}

\section{Psychohygienische und psychosomatische Aspekte}

Herausgegeben von

Primarius Dr. Hans Zimprich

Springer-Verlag Wien GmbH 1980

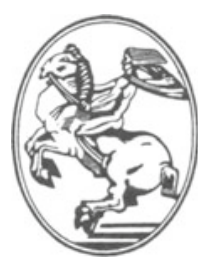


Primarius Dr. Hans Zimprich,

Vorstand der Kinderinternen Abteilung mit Psychosomatik, Wilhelminenspital, Wien, Österreich.

Das Werk ist urheberrechtlich geschützt.

Die dadurch begründeten Rechte, insbesondere die der Übersetzung, des Nachdruckes, der Entnahme von Abbildungen, der Funksendung, der Wiedergabe auf photomechanischem oder ähnlichem Wege und der Speicherung in Datenverarbeitungsanlagen, bleiben, auch bei nur auszugsweiser Verwertung, vorbehalten.

(C) 1980 by Springer-Verlag Wien

Ursprünglich erschienen bei Springer Vienna 1980.

CIP-Kurztitelaufnahme der Deutschen Bibhothek

Kind und Umwelt: psychohygien. u. psychosomat. Aspekte / hrsg. von Hans Zimprich. - Wien, New York: Springer, 1980.

(Pädiatrie und Pädologie: Suppl; 6)

NE: Zimprich, Hans [Hrsg.] 


\section{Vorwort}

Das Buch „,Kind und Umwelt“ ist als Sammelband nach drei Symposien des Vereines „Kind-Familie-Umwelt“ entstanden. Der Verein setzt sich die Verbreitung psychohygienischen Wissens in praktischer wie in wissenschaftlicher Weise zur Aufgabe. Der Band enthält dementsprechend in seinen beiden Teilen sowohl psychohygienische als auch psychotherapeutische Erfahrungsberichte namhafter Experten auf diesen Gebieten, wobei für den Leser der multidisziplinäre Ansatz von besonderem Interesse sein wird. Da der praktisch tätige Kinderarzt, für den dieses Buch in erster Linie geschrieben wurde, für seine Arbeit einen großen Bedarf an Wissen in solchen Grenzgebieten hat, wird er psychoanalytische, pädagogische und von Psychologen geschriebene Kapitel als Ergänzung seiner medizinischen Aspekte wertvoll finden, während aus anderen Gebieten kommende Leser wie Sozialarbeiter, Lehrer und andere mit Kindern oder in Familien arbeitende Berufsgruppen medizinisch-psychosomatische Kapitel von besonderem Interesse finden können. Die Auswahl der Kapitel wurde im Blickpunkt der Komplexität getroffen, um der Weite des Wissensgebietes entsprechend eine möglichst umfassende Darstellung geben zu können und gleichzeitig praktische Hilfe für diejenigen Kinder, die an psychosomatischen Krankheiten leiden oder nahe daran sind, zu erkranken.

Wien, im Dezember 1979

H. Zimprich 


\section{Inhaltsverzeichnis}

Verzeichnis der Mitarbeiter . . . . . . . . . . . . . . . . . . VII

\section{Editorial}

Berner, P.: Psychohygiene im Kindesalter $\ldots \ldots \ldots \ldots \ldots \ldots$

Psychohygienisch-pädagogische Aspekte zur

Prävention psychosomatischer Erkrankungen

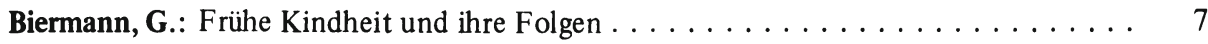

Schwarzmann, Julia: Psychoanaly tische Gedanken zu Erziehungsfragen . . . . . . . 25

Buchinger, K.: Die Bedeutung eines Intimbereiches in der Erziehung . . . . . . . 37

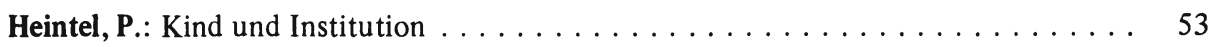

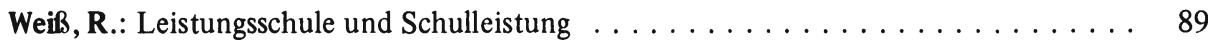

\section{Psychosomatisch-pädiatrische Aspekte}

Zweymüller, E.: Wandlung pädiatrischer Schwerpunkte . . . . . . . . . . . . 101

Spiel, W.: Uberlegungen zum Problem der Psychosomatosen und anderer frühkindlicher

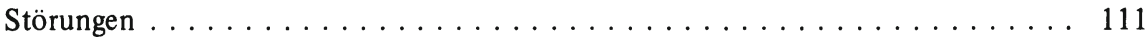

Ringel, E.: Die psychosomatische Grundhaltung des Arztes . . . . . . . . . . 121

Zimprich, H.: Behandlungskonzepte und -resultate bei psychosomatischen Erkrankungen

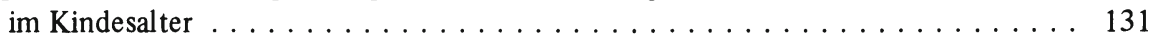

Günther, K. B.: Aspekte gemeinsamer Verhaltensqualitäten und Interaktionen psychosomatisch Kranker . . . . . . . . . . . . . . . . . . . . . . . . . . . . . 199

Scheer, P.: Therapieverfahren in der Psychosomatik. Indikation und Anwendung . . . . 207 


\section{Verzeichnis der Mitarbeiter}

Berner, Univ.-Prof. Dr. P., Vorstand der Psychiatrischen Universitätsklinik, Währinger Gürtel 74-76, A-1090 Wien, Österreich.

Biermann, Univ.-Prof. Dr. G., Bäumlstraße 22, D-8031 Puchheim, Bundesrepublik Deutschland.

Buchinger, Dr. K., Institut für Tiefenpsychologie und Psychotherapie der Universität (Vorstand: Univ.-Prof. Dr. H. Strotzka), Währinger Gürtel 74-76, A-1090 Wien, Österreich.

Günther, Dr. K. B., Kinderinterne Abteilung mit Psychosomatik, Wilhelminenspital der Stadt Wien, Montleartstraße 37, A-1171 Wien, Österreich.

Heintel, Univ.-Prof. Dr. P., Vorstand des Instituts für Philosophie der Universität für Bildungswissenschaften, Universitätsstraße 65-67, A-9020 Klagenfurt, Österreich.

Ringel, Univ.-Prof. Dr. E., Leiter der psychosomatischen Abteilung der Psychiatrischen Universitätsklinik, Währinger Gürtel 74-76, A-1090 Wien, Österreich.

Scheer, Dr. P., Kinderinterne Abteilung mit Psychosomatik, Wilhelminenspital der Stadt Wien, Montleartstraße 37, A-1171 Wien, Österreich.

Schwarzmann, Dr. Julia, Lothringerstraße 18, CH-4056 Basel, Schweiz.

Spiel, Univ.-Prof. Dr. W., Vorstand der Universitätsklinik für Neuropsychiatrie des Kindes- und Jugendalters, Währinger Gürtel 74-76, A-1090 Wien, Österreich.

Weiß, Univ.-Prof. Dr. R., Vorstand des Instituts für Erziehungswissenschaften der Universität, Innrain 30, A-6020 Innsbruck, Österreich.

Zimprich, Primarius Dr. H., Vorstand der Kinderinternen Abteilung mit Psychosomatik, Wilhelminenspital der Stadt Wien, Montleartstraße 37, A-1171 Wien, Österreich.

Zweymüller, Univ.-Prof. Dr. E., Vorstand der Universitäts-Kinderklinik, Währinger Gürtel 74-76, A-1090 Wien, Österreich. 